\title{
Aneurysm brain with cavernous sinus dissection: case report
}

\begin{abstract}
Aneurysms in the cavernous segment of internal carotid artery correspond from $2 \%$ to $9 \%$ of all intracranial aneurysms and are completely extradural, presenting a low risk of progressing to a spontaneous subarachnoid hemorrhage, thus having a relatively low mortality and morbidity. Its cause may be idiopathic, traumatic, iatrogenic or infectious. The symptomatology is associated with the size of the aneurysm and thrombosis within, may be asymptomatic or bleeding, meningeal signs, focal deficit, confusion, drowsiness, especially severe headache, which may be coming from the compression of cranial nerves present inside the cavernous sinus with retro-orbital pain or changes in extra ocular motility. The dissection of the breast is challenging and can injure these nerves. The authors present the case of a woman with unruptured aneurysm in unusual segment by topography, with indicative injury to cranial nerves.
\end{abstract}

Keywords: aneurysm unruptured, cavernous sinus, oculomotor nerve
Volume 5 Issue I - 202 I

\author{
Roseane Lucena Marquez,' Ana Luisa Sabino \\ de Campos Faria,' Pedro Henrique Essado \\ Maya, ${ }^{2}$ José Edison da Silva Cavalcante, ${ }^{3}$ \\ Joubert Borges de Almeida Junior ${ }^{2}$ \\ 'Doctor graduated from Pontifícia Universidade Católica de \\ Goiás, Goiânia, Goiás, Brazil \\ ${ }^{2}$ Resident Doctor at the Neurosurgery Service of Hospital de \\ Neurologia Santa Mônica- Hospital das Clínicas / Universidade \\ Federal de Goiás, Goiânia, Goiás, Brazil \\ ${ }^{3}$ Chief Neurosurgeon of the Neurosurgery Service of \\ Hospital de Neurologia Santa Mônica - Hospital das Clínicas / \\ Universidade Federal de Goiás, Goiânia, Goiás, Brazil
}

Correspondence: Roseane Lucena Marquez. Address:Avenida Bela Vista Quadra 0I Lote 08 Jardim Progresso, Aparecida de Goiânia, Goiás, Brasil. Zip code: 74.9|।-720. Phones for contact: (62) 99285-4004 and (62) 3282-3113. Email roseanelcmq@gmail.com.

Alternative email roseane.-.Imq@hotmail.com

Received: December 22, 2020 | Published: January 07, 2021

\section{Introduction}

Aneurysm is a reflex of arterial dilatation that results in weakening of the arterial wall..$^{5}$ It can be sacculate, fusiform or dissecting, being possible in any intracranial location. ${ }^{5}$ Aneurysms in the cavernous segment of the internal carotid artery account for $2 \%$ to $9 \%$ of all intracranial aneurysms and are completely extradural and present a risk of developing spontaneous subarachnoid hemorrhage (SAH), thus having a lower morbidity and mortality., Its etiology may be idiopathic, traumatic, iatrogenic or infectious. ${ }^{3}$ The clinical manifestations of the aneurysm are associated with the size of the aneurysm and thrombosis in the interior, and may be asymptomatic or with bleeding, meningeal signs, focal deficit, mental confusion and somnolence. ${ }^{5,1}$ However, the most prevalent symptom is a headache that can result in paralysis of the oculomotor nerve, sometimes the presentation of retro-orbital or changes in extrinsic ocular motricity. ${ }^{5,2}$ Headache is reported by $18 \%$ of patients with non-ruptured cerebral aneurysm. ${ }^{4}$ Approximately patients have an abnormality of one or more cranial nerves that travel through the cavernous sinus (III, IV, $\mathrm{V} 1, \mathrm{~V} 2$, and VI). ${ }^{7}$ The sinus dissection is challenging and may injure the cranial nerves not present within the cavernous sinus. ${ }^{4}$ This study aims to report the case of a patient with an unmarked aneurysm in the segment not usual for the topography, with indication of the cranial pairs, attended at Hospital Santa Mônica, in Aparecida de Goiânia Goiás, literature review on the subject.

\section{Methods}

The data referring to the description of the clinical case were collected from the patient's medical record, with the authorization of the patient. The study met the ethical criteria of Resolution 466/12 of the Conselho Nacional de Saúde and was approved by the Comitê de Ética em Pesquisa of Hospital de Neurologia Santa Mônica. The participant was invited to participate as a volunteer in the research and was clarified about the objectives, stages and relevance of this research, as well as clarification of any doubts about it. By agreeing to participate in this, the participant signed the Free and Informed Consent Form (FICF), present in two ways of equal value. In addition, a search was made for indexed journals in the Scielo (Scientific Electronic Library Online), PubMed (US National Library of Medicine) and Academic Google databases using the English equivalents: cavernous aneurysm, cavernous sinus dissection, oculomotor nerve, internal carotid artery. Using the time cut from 2004 to 2014, 6 more pertinent articles were selected for the construction of the theoretical foundation.

\section{Case report}

Female patient, 51 years old, brown, born in Juazeiro, Ceará. There was a month with progressive headache, pulsatile type in fronto-temporal region and right retro-ocular. In a few days, the right palpebral ptosis and hyperalgesia in V1 topography were added to the headache. Use of analgesics without clinical improvement ("the pain was so intense that she considered the possibility of suicide"). Absence of history of aneurysm and systemic diseases in the family. Deny contraceptive use. At physical examination: regular general condition, painful fascia, afebrile, atypical gait, eudychokinesis, right palpebral ptosis, as shown in Figure 1, consensual photoreagent, right mydriasis, as shown in Figure 2, hyperalgesia in V1 topography, strabismus divergent, as shown in Figure $3 \& 4$, and photophobia. Angiography confirmed the presence of sacculate aneurysm of the posterior communicating segment of the right internal carotid artery, as shown in Figure 5, also confirmed by magnetic resonance and magnetic resonance angiography, as shown in Figure $6 \&$ 7. During the surgical procedure it was confirmed that the aneurysm dissected 
the cavernous sinus. However, it was observed that, instead of the lateral aneurysm in contact with the third cranial pair in the temporal, which is the habitual one, it made a medial orientation in contact with this in the cavernous sinus, due to the conformation of the aneurism in the form of hammer. ${ }^{6}$ This can also be justified by the fact that the posterior communicating artery in this case has no fetal pattern and the caliber of it is inferior to the posterior cerebral artery branch P1. ${ }^{6}$ In this way, it leaves the internal carotid artery in a more medial orientation. ${ }^{6}$ In the postoperative period there was an improvement in pain, but palpebral ptosis on the right, divergent strabismus, consensual photoreagent, fixed right mydriasis and photophobia persisted. Control angiography showed absence of residual aneurysm in the posterior communicating segment of the right internal carotid artery. Figure 8 shows the clipping of the region involved in the postoperative period.

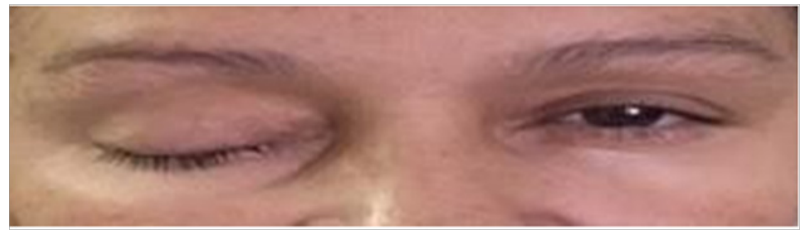

Figure I Right palpebral ptosis.

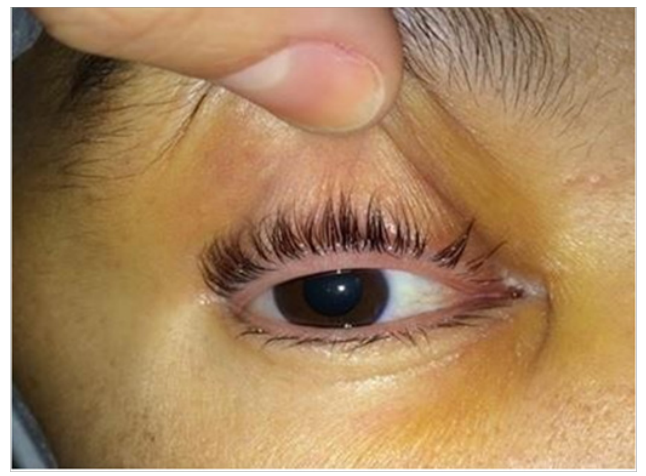

Figure 2 Right fixed mydriasis.

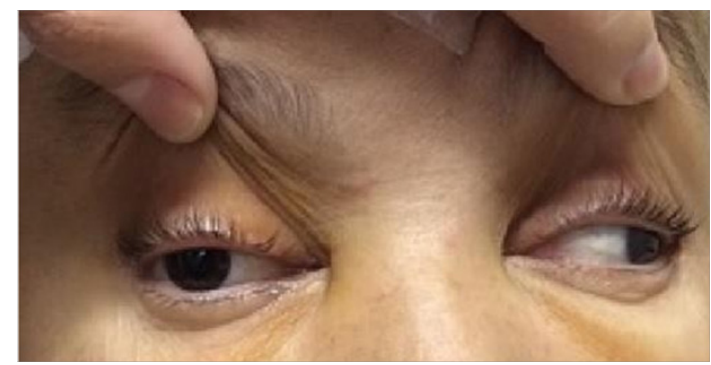

Figure 3 Divergent strabismus on the left.

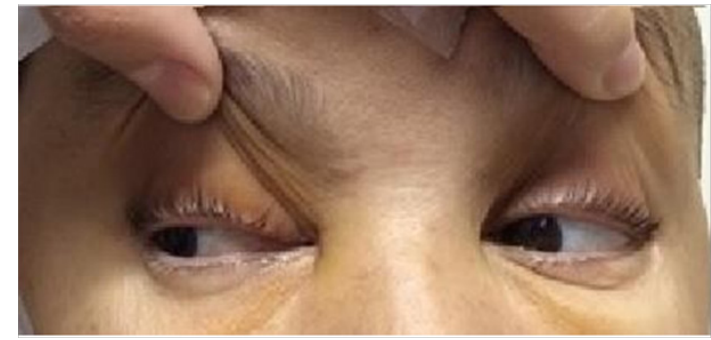

Figure 4 Patient's eye movement confirming correlated strabismus.

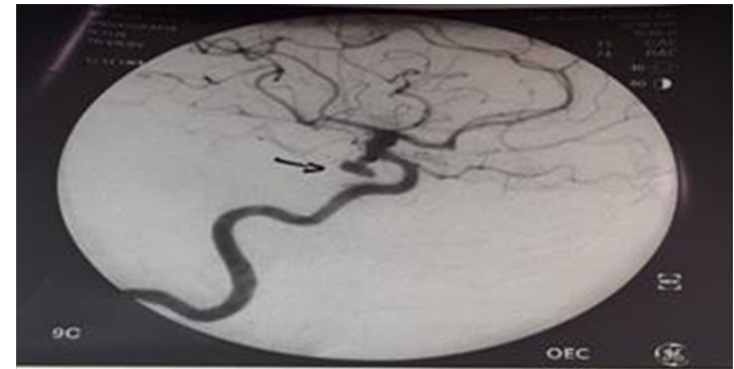

CArótioa (D) Peracil

Figure 5 Angiography showing aneurysm in the right internal carotid artery.

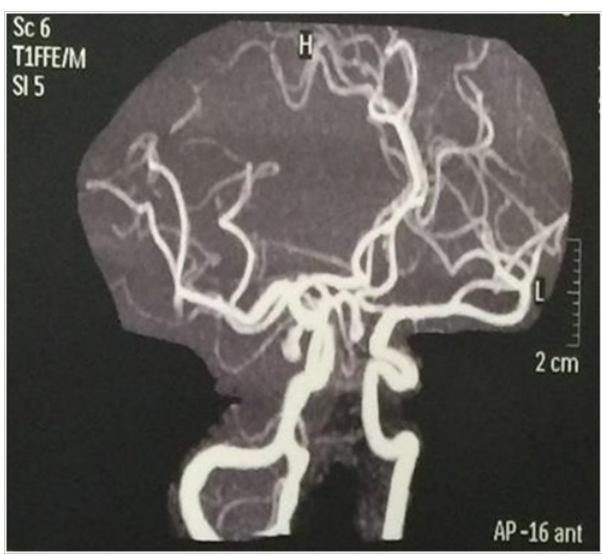

Figure 6 Magnetic resonance angiography showing correlated aneurysm.

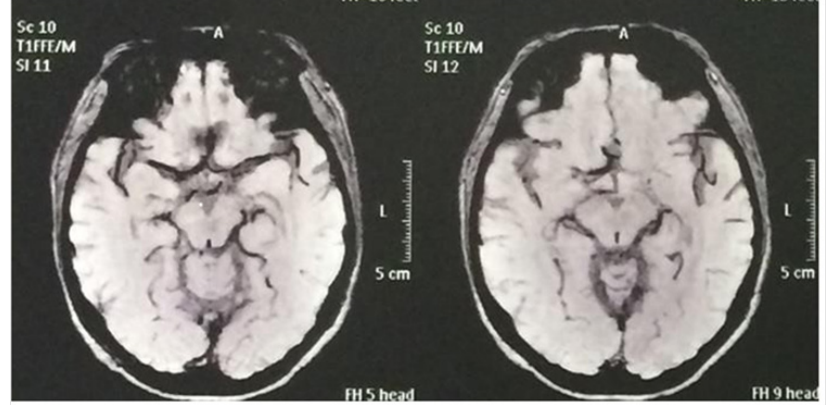

Figure 7 Preoperative showing anatomical region of the cavernous sinus and right internal carotid Artery.

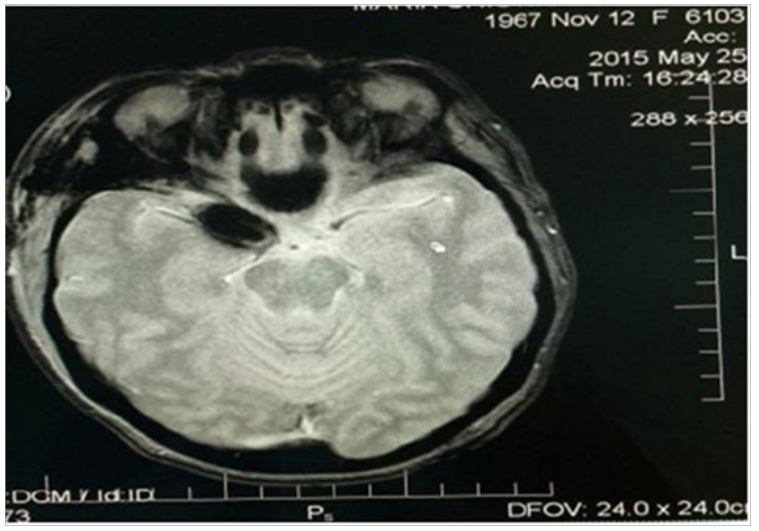

Figure 8 Post-operative show in. 


\section{Conclusion}

Removal of lesions, especially aneurysms, by dissecting the cavernous sinus is challenging in the field of neurosurgery in view of the difficulty of preserving the neural and vascular structures during the surgical procedure, besides the existence of few scientific papers detailing this theme, which makes this a case report of great importance for scientific knowledge. ${ }^{4}$

\section{Acknowledgments}

None.

\section{Financing}

The costs for carrying out the research were borne by the researchers themselves.

\section{Conflicts of interests}

The authors declare that they have no conflict of interest.

\section{Patient consent}

The patient has consented to the submission of the case report for submission to the Journal.

\section{References}

1. Aguiar GB, Neto DGG, Silva JMA, et al. Advances in Endovascular Treatment of Cavernous Internal Carotid Artery Aneurysms. J Bras Neurocirurg. 2014;25(1):46-52.

2. Ambekar S, Madhugiri V, Sharma M, et al. Evolution of management strategies for cavernous carotid aneurysms: A Review. World Neurosurg. 2014;82(6):1077-1085.

3. Eddleman CS, Hurley MC, Bendok BR, et al. Cavernous carotid aneurysms: to treat or not to treat? Neurosurg Focus. 2019;26(5):E4.

4. Olesen J, Bousser MG, Diener FHC, et al. Headache Classification Subcommittee of the International Headache Society. The International Classification of Headaches Disorders. $2^{\text {nd }}$ edition. Brazilian Headache Society. São Paulo: Editorial Lute. 2006. p. 1-211.

5. Pierobon MR. Anatomo-radiological classification of posterior communicating artery aneurysms. Porto Alegre: Universidade Federal do Rio Grande do Sul. 2012.

6. Rhoton. Skull - Anatomy and Surgical Access. Publisher Dilivros. $1^{\text {st }}$ edn. 2003. p. 752.

7. Tedeschi H, Oliveira E, Tzu WH. Surgical treatment of lesions in the cavernous sinus region. Neurosurgery Society of the State of São Paulo. São Paulo: Lemos Editorial. 2004. 